\title{
El clientelismo en el modelo politico venezolano: un análisis preliminar
}

\author{
Rexene A. Hanes de Acevedo
}

La crisis financiera que estalló en Venezuela el famoso "viernes negro", 18 de febrero de 1983, conllevó a una introspección nacional sobre la vigencia o agotamiento del modelo politicoeconómico imperante en el país desde 1958. "Se agotó el modelo" se ha convertido en lugar común en los artículos de prensa y en los discursos políticos (incluso en los académicos). Sin embargo, esta discusión nos parece, además de imprecisa, poco fecunda.

Tal vez debiéramos aclarar, de una vez, lo que es, para los propósitos del presente trabajo, un modelo. Cuando hablamos del modelo político venezolano, no nos referimos a una representación matemática de las relaciones políticas, ni tampoco al proyecto político de tal o cual personaje o sector de la sociedad venezolana. Nos referimos fundamentalmente a una abstracción de ciertas variables cruciales, y las interrelaciones entre ellas, que nos puede ayudar a comprender el funcionamiento del sistema político y ofrecernos algunos indicios de posibles cambios a la luz de la crisis actual.

Pero cualquier intento de construir, en este sentido, un modelo del sistema político venezolano está repleto de peligros: por ejemplo, ¿cuáles son las variables cruciales? Por esto nos parece prudente recordar una advertencia que hace el destacado historiador venezolano Germán Carrera Damas en su libro Una nación llamada Venezuela y que es muy apropiada para nuestro tema:

dado el conocimiento insuficiente de los "invariantes" en el cambio; dada la insuficiencia de la información disponible, $y$, por último, dada la incipiencia del sistema científico nacional, todo esfuerzo de penet ración prospectiva, no ya del conjunto de la sociedad sino de cualquiera de sus áreas, tropieza con tan serios obstáculos que el ir más allá de un planteamiento prudencial, o más bien del señalamiento de algunas tendencias, puede resultar pura y simple adivinación.

Si usamos el término "modelo político" con las debidas precauciones, el "agotamiento" o no del modelo no es tan importante como lo es un comprensión adecuada de las variables involucradas y sus interrelaciones. Ha habido algunos esfuerzos en este sentido, ${ }^{1}$ pero en todos, algunos factores, como por ejemplo el clientelismo político, tan omnipresente en la sociedad venezolana, brillan por su ausencia. Sin menoscabo de la importancia de otros factores, nos proponemos en este trabajo indagar respecto a la funcion del clientelismo en el modelo

1 Véase entre otros, Diego Bautista Urdaneja, "El sistema político o cómo funciona la máquina de procesar decisiones", en M. Naim y R. Piñango (eds.), El caso de Venezuela: una ilusion de armonta, Caracas, Ediciones IESA, 1984, p. 228-257; Daniel Levine, "Venezuelan Politics: Past and Future", en Robert D. Bond (ed.), Contemporany Venezuela and its Role in Intermational Affairs, Nueva York, New York University Press, 1977, p. 7.44 y Manuel Suzzarim Baloa, Romulo Betancourt, proyecto de modemización, Caracas, Editorial Ateneo, 1981. 
político venezolano y sus posibles tendencias al cambio en la presente situación de estrechez financiera.

Este análisis del modelo político venezolano parte de una premisa: que el problema más importante que encaran los gobiernos de los países dependientes es el control sobre el proceso de desarrollo. ${ }^{2} \mathrm{De}$ esta importancia da fe la Constitución venezolana de 1961, que estabece que, a fin de lograr la justicia social y la soberanía economica del país, "El Estado promoverá el desarrollo económico y la diversificación de la producción..." (Artículo 95) y le da facultades para "dictar medidas, para planificar, racionalizar y fomentar la producción, y regular la circulación, distribución y consumo de la riqueza, a fin de impulsar el desarrollo económico del país" (Artículo 98). También da fe, aunque de forma más sutil, de otro hecho que forma parte de la premisa original: puesto que el poder que puede ejercer el gobierno sobre los factores externos es poco o indirecto, el control sobre los procesos políticos y económicos internos viene a ser uno de los objetivos primordiales de esos gobiernos. El clientelismo político proporciona un cierto grado de control sobre las actividades del cliente, como elemento inseparable del mismo gobierno y, por ende, sería un mecanismo importante para lograr ese objetivo.

Antes de indagar sobre las implicaciones de esta premisa para el análisis del clientelismo en el modelo político venezolano, es necesario primero definir el concepto del clientelismo político y repasar brevemente su desarrollo histórico en América Latina.

Existe un consenso general en la literatura en lo referente a la naturaleza de los lazos políticos clientelistas tradicionales en cuanto que éstos se basan en el prototipo de las relaciones entre el terrateniente y el peón, caracterizadas éstas por el gran latifundio y las extremas desigualdades de status. ${ }^{3}$ A partir de este prototipo, las relaciones clientelistas se pueden definir como transacciones de intercambio de bienes y servicios no comparables entre personas que tienen status y recursos desiguales. ${ }^{4}$

Varios puntos destacan en esta definición: en primer lugar, los lazos clientelistas son lazos verticales que vinculan a personas de diferentes status y recursos. A medida que estas relaciones se extiendan más en la sociedad y se liguen entre sí, formando redes con fuertes lazos verticales y lazos horizontales débiles o insignificantes, se hará más difícil el reconocimiento de los intereses comunes de clase y la formación de organizaciones cuya base sean las clases sociales.

En segundo lugar, los lazos clientelistas se basan en última instancia en las disparidades de poder. Y, como ocurre con todas la relaciones de poder, los lazos clientelistas son, en mayor o menor grado, recípro-

2 Véase Richard N. Adams, The Second Sowing: Power and Secondary Development in Latin America, San Francisco, Chandler, 1967.

3 Véase Steffen Schmidt et al., Friends, Followers and Factions: a Reader in Political Clientelism, Berkeley, University of California Press, 1977.

4 Kene Lemarchand y Keith Legg, "Political Clientelism and Development", Comparative Politics, vol. 44, núm. 2, enero, p. 149-178, 1972, p. 151; John Duncan Powell, "Peasant Society and Clientelist Politics", American Political Science Review, vol 64, num 2, 1970, p. 413, y James Scott, "Patron-Client Politics and Political Change in Southeast Asia", American Political Science Review, vol. 66, núm. 1, marzo 1972, p. 92-95.

5 George M. Foster, "The Dyadic Contract; A Model for the Social Structure of a Mexican Peasant Village", en S. Schmidt et al, op. cit, p. 27; Richard Sandbrook, "Patrons, Clients, and Factions: New Dimentions of Conflict Analysis in Africa", Canadian Joumal of Political Science, vol. 5, núm. 2, marzo 1972, p. 207 y James Scott, op. cit, p. 91. 
$\cos ^{6}{ }^{6}$ El grado de reciprocidad depende, en gran parte, del monopolio de los recursos y las asimetrías de poder involucradas. En todo caso, los intercambios son desiguales.

Tercero, estos intercambios recíprocos no son contractuales; no obligan legalmente. Así, se puede explicar el marcado énfasis en las relaciones cara a cara, en el personalismo y el afecto en las relaciones clientelistas tradicionales, con el fin de asegurar el cumplimiento de estas obligaciones.

Finalmente, el intercambio de bienes y servicios no comparables se presta fácilmente a la difusión de las transacciones, que es característica de las obligaciones clientelistas. Es precisamente esta característica la que proporciona a esos lazos su enorme flexibilidad y elasticidad para adaptarse a las circunstancias cambiantes y así perdurar, manteniendo intactas sus características básicas.

También existe bastante consenso alrededor de los orígenes del clientelismo. En general, se puede decir que las relaciones clientelistas surgen como respuesta a dos condiciones: La primera es la escasez de recursos, tanto naturales como sociales, que a su vez están desigualmente distribuidos. Pero aún cuando ésta es una condición necesaria, no es suficiente para explicar el origen y la persistencia de las relaciones clientelistas.

La inseguridad proveniente del entorno físico y social constituye la segunda condición necesaria para el origen y continuación de las relaciones clientelistas. Esta inseguridad puede tomar muchas formas. Puede amenazar la misma supervivencia o la estabilidad en el trabajo, o simplemente el ascenso en el mismo.

El papel del Estado también entra en los cálculos de la inseguridad proveniente del entorno. Donde la penetración del Estado es débil, donde no existen (o tienen fallas) las funciones estatales de bienestar social, donde el Estado no puede proporcionar protección a la vida, a la propiedad y a la riqueza, surgirán otros mecanismos para cumplir con estas funciones. En algunos casos, extensos sistemas clientelistas pueden convertirse incluso en equivalentes funcionales del Estado.

En cualquier caso, las redes clientelistas tienen ramificaciones políticas importantes, capaces de complementar las funciones del Estado. Dicho de otra manera, actúan como "una especie de relleno plástico, que puede llenar las hendiduras cambiantes de las estructuras sociales y servir como una especie de cemento social general". Por su naturaleza intersticial, estos sistemas de clientela sirven para llenar los resquicios dejados por la penetración incompleta del Estado en la sociedad.

Las variables asociadas con las relaciones clientelistas tienden a agruparse en patrones reconocibles a través de las diferentes etapas del desarrollo y a cambiarlos en respuesta a cambios societarios. Básicamente, existen dos tipos de clientelismo político: 1) el tradicional y 2) el corporativo.

Las relaciones clientelistas tradicionales se caracterizan por un alto grado de personalismo, por la falta de fuentes alternas de patronazgo, por tener los patronos casi un monopolio sobre los recursos, por su larga duración, y por el carácter netamente particularista de los favores pedidos y recibidos. La base de los recursos disponibles en los sistemas tradicionales de patronazgo es bastante estable y limitada, dependiendo 
principalmente de la riqueza del patrón, por una parte, y de la mano de obra y lealtad del cliente, por la otra. En otras palabras, la expansión del clientelismo se da en función de la limitación de los recursos controlados directamenrte por el patrón.

Este tipo de clientelismo predominó en América Latina durante el periodo colonial y el primer siglo de la Independencia, épocas en las que las sociedades latinoamericanas eran esencialmente agrarias, con extensas propiedades relativamente aisladas y autónomas, y caracterizadas por una penetración muy débil del Estado. La última parte del siglo XIX y la primera mitad del siglo XX presenciaron muchos cambios sociales que más tarde se reflejarían en los aspectos variables de los sistemas clientelistas. Con los cambios socioeconómicos, el clientelismo tradicional también se transformó en lo que en otra parte hemos caracterizado como el clientelismo corporativo.

Las transformaciones ocurridas en la economía y en la sociedad beneficiaron considerablemente a la burguesía nacional incipiente y a los sectores medios emergentes. La extrema escasez de recursos, tanto materiales como no materiales, siguió siendo la característica distintiva de los sectores populares. La migración interna sobrepasó en mucho la capacidad de la industria para absorber el flujo de inmigrantes a las ciudades, lo que creó una intensa competencia interna por empleo, tanto en la industria como en el sector terciario, y así las condiciones de vida de los recién llegados siguieron siendo precarias. En medio del cambio social, la inseguridad se exacerba en lugar de aliviarse y, en consecuencia, aumenta la necesidad de buscar mecanismos sociales que la reduzcan.

Mientras en las zonas rurales el señor agrarista continuaba desempeñando un papel importante, en las áreas urbanas se sentía también la necesidad de un patrón. La concentración en las zonas urbanas de una población numerosa en búsqueda de un patrón, en conjunción con sectores medios emergentes que buscaban poder político y una crisis económica que demostraba la vulnerabilidad del crecimiento hacia afuera, condujo al surgimiento del populismo como una forma de acabar con la dominación política de la oligarquía terrateniente.

En su búsqueda de poder, los sectores medios necesitaban la fuerza electoral inherente a las organizaciones de masas, pero su éxito dependía en gran parte de su habilidad para controlar y manipular esa activación política. Así, el control surge como un elemento principal de la incorporación de los sectores populares a la lucha por el poder político. El clientelismo corporativo, de base masiva (ya no individual), proporcionó justamente tal mecanismo de activación y control de la participación política popular y el populismo fue su expresion.

En el clientelismo corporativo, grupos dentro del Estado o relacionados con él entraron en una relación de intercambio de bienes y servicios con algunos sectores de las clases populares. Los bienes y servicios son de tipo no comparables (generalmente, prebendas económicas por apoyo político). Si bien, en este caso, los actores no son necesariamente individuos que representan intereses individuales, poseen definitivamente el status y los recursos desiguales (status y poder del Estado versus los de las clases populares, recursos del Estado versus recursos individuales, etc.). Aunque los intercambios son recíprocos, siguen siendo desiguales y las relaciones resultantes involucran controles y limitaciones sobre las actividades de los clientes.

A medida que el Estado penetre esferas adicionales de la sociedad y se concentren en él mas recursos y poder, simultáneamente va adqui- 
riendo mayor importancia el aspecto de control inherente al clientelismo. La base fundamental del clientelismo es el intercambio de bienes y servicios no comparables; sin embargo, a medida que la relación se haga más institucionalizada y más duradera, las transacciones de intercambio pueden hacerse menos iguales y menos importantes para el mantenimiento de la relación. Y a menudo es lo que ocurre. En un primer momento, cuando se buscaba fortalecer el poder del Estado y consolidar el dominio de los nuevos grupos, el aspecto de intercambio tuvo primacía sobre el aspecto de control, aunque nunca se ignoró éste. Sin embargo, al continuar la relación, el Estado (o los grupos asociados a él) va ligando cada vez más estrechamente a los grupos de clientela. A medida que la relación se institucionaliza y se consolida el poder del Estado, la clientela se hace más dependiente y el enfasis sobre el intercambio cede el paso al énfasis sobre el control.

Este breve repaso del clientelismo y los cambios que ha sufrido, nos proporciona un marco general para entrar a analizar el modelo político venezolano en vigencia desde 1958. El modelo betancouriano se podría considerar un modelo populista o de clientelismo corporativo, con ligeras variantes. También se podría calificar como una democracia subsidiada, para resaltar la íntima relación entre la capacidad económica del Estado y su capacidad política.

La estabilidad política del sistema democrático venezolano está determinada por la capacidad del Estado para mantener un cierto consenso social. Pero ese consenso social no se centra en la adhensión al sistema democrático en sí. David Eugene Blank afirma que "De hecho, la actitud de las masas venezolanas hacia la democracia es, en el mejor de los casos, de una gran indiferencia." También el estudio del CENDES-MIT encontró muy poco compromiso con los valores democráticos entre los séctores urbanos, con excepción de las elites. Las elites sí demuestran tener cierto compromiso con el sistema democrático, si bien no tanto con los valores democráticos (muchos, en el estudio del CENDES tendían a ver al pueblo como una carga o un estorbo para lograr el desarrollo nacional).

Un ejemplo de la poca internalización de la democracia se puede ver en la anécdota de la Convención Nacional de Acción Democrática en 1966, cuando se discutía la tesis política de AD. Demetrio Boersner había planteado que " $\mathrm{AD}$ acrece su acervo doctrinario en la corriente universal del pensamiento socialista." Y Rómulo Betancourt le contesta en una carta desde el exterior: "AD no requiere remoquetes ideológicos". La idea de Betancourt parece haber sido que no se adquiere compromiso con valores democráticos si no se proporcionan mejoras en el nivel de vida de la población mediante prebendas materiales, tales como la construcción de escuelas, carreteras, inversiones en la industria, etcétera.

Sin embargo, Germán Carrera Damas plantea que la realización del Estado democrático liberal ha sido una meta constante de las elites venezolanas desde el siglo pasado. Y que todas las elites más avanzadas desde 1936 se han movido dentro de esta "trampa ideológica" de aceptar la identificación entre nación y Estado liberal democrático planteado por la clase dominante del siglo XIX. Según él, se ha logrado adaptar ese proyecto nacional a nuevas realidades, pero hasta ahora ningún grupo ha logrado formular un nuevo proyecto nacional que exprese la coyuntura sociohistórica. Por ende, todos se mueven dentro de la institucionalización del Estado democrático liberal. No obstante, no se puede extraer este proyecto político “democratizador" del proyecto 
"socioeconómicamente modernizador". Parece haber cierto consenso, no únicamente entre las elites, sino entre la población en general, de que los gobiernos democráticos han resultado más eficaces que los militares en promover el proyecto modernizador. En este entrelazamiento entre la institucionalización del Estado democrático liberal y la búsqueda del desarrollo șocioeconómico es donde se percibe con mayor claridad el compromiso nacional con el sistema democrático.

Tal vez de esta forma se expliquen mejor los resultados aparentemente contradictorios con los estudios arriba referidos, encontrados por Baloyra y Martz en su análisis de las actitudes políticas en Venezuela. Según estos autores, los obreros demuestran igual o mayor compromiso con los valores democráticos que personas de status más alto, junto con una mayor militancia en los partidos políticos. Sin embargo, los sectores de menor status también tienden a ser más "críticos", "cínicos" y a tener menos sentido de eficacia política que sus compatriotas de mayor rango socioeconómico. Estos datos son comprensibles y no contradicen neceseriamente los estudios anteriores si analizamos las relaciones que mantienen los partidos políticos con las organizaciones populares, en términos de clientelismo político. El análisis nos sugeriría que el grado de compromiso de los sectores populares, con los valores democráticos depende en gran parte de la capacidad del Estado democrático de ofrecer las prebendas necesarias para perpetuar la relación.

En resumen, parece que los valores democráticos no están muy interiorizados en ningún sector de la sociedad venezolana, aunque las elites (económicas, políticas, etc.) parecen tener más compromiso con el sistema democrático como una forma de lograr el desarrollo nacional, lo que demuestra en términos bastante francos que la capacidad de mantener la adhesión de los diferentes sectores al sistema democrático se centra en la capacidad económica del Estado, en su capacidad de garantizar servicios, empleo e inversiones. Betancourt entendía claramente esta interrelación, hecho que se demuesta con la proposición que hizo a los dirigentes empresariales y sindicales en la campaña electoral de 1958 de "un pacto de desarrollo económico y paz laboral como camino para consolidar el régimen de las instituciones democráticas". Por algo se suele denominar al sistema actual como el "modelo betancouriano".

Pero la capacidad económica del Estado depende del petróleo. El petróleo domina el ritmo económico interno, que a la vez determina el ritmo del sistema político, o sea el grado de consenso social en torno al sistema político. Basándose en la renta petrolera, que ha venido fortaleciendo el poder económico del Estado durante más de cincuenta años, y que para 1961 convierte al "Estado, constitucionalmente en rector de la vida económica y social", se constituye el modelo político venezolano actual. Debido a los grandes requerimientos de recursos financieros que implica este modelo, el gobierno venezolano empezó a endeudarse sistemáticamente, a partir de 1975 aproximadamente, para poder mantenerlo y fortalecerlo. La combinación de este factor con la súbita caída de los precios petroleros llevó a la actual crisis.

Para entender en qué consiste el modelo de la "venedemocracia", es necesario primero retroceder en el tiempo a la única otra experiencia democrática venezolana de este siglo. Una de las razones por la que fracasó el intento democrático del trienio 1945-1948 fue la falta de control (o la percepción de la falta de control) sobre el proceso de incorporación de nuevos sectores al juego político. 
La política durante el trienio fue marcada por un conflicto amplio, amargo y $\sin$ cesar, que culminó en un golpe militar y una década de represión brutal. La experiencia de estos años marcó profundamente a los líderes políticos venezolanos. Se convencieron de que su propia falta de controlar y canalizar el conflicto político había abierto la puerta al golpe militar. Aprendieron, además, que el liderazgo político involucra algo más que el apego a la ideología y al programa; la conciliación y la negociación se convirtieron en valores políticos claves.?

Pero la reconciliación y negociación con otras elites sería imposible si los líderes políticos no pudieran controlar las demandas populares de sus propias organizaciones. Así surge la necesidad de control como parte de la concepción política a partir de 1958. El mismo autor nota que, desde la instalación del nuevo régimen democrático,

los líderes políticos parecer estar constantemente volteando hacia atrás. Se comportan como si las instituciones políticas fueran a sufrir un colapso en cualquier momento... El recurso de conflictos ilimitados ha hecho que muchos líderes manejen la política con extremo cuidado. ${ }^{8}$

Naim y Piñango señalan el mismo fenómeno cuando caracterizan al conflicto abierto como "el eslabón perdido en la evolución venezolana reciente" y "la evasión del conflicto como norma inviolable" del sistema.

En otras palabras, hay que controlar los niveles de conflicto. En gran parte, aunque parezca contradictorio, los partidos políticos han venido a cumplir esta función. Uno podría aceptar la afirmación de Blank de que el sistema político actual es más el resultado del desarrollo del sistema partidista que de la Constitución de 1961.

Una de las primeras expresiones de lo que iba a ser ese modelo político se encuentra en el Pacto de Punto Fijo, que garantizaba acceso a la formulación de políticas a los partidos y a los grupos de presión y el cumplimiento de un programa mínimo que asegurara cierta estabilidad política. La reforma agraria, la expansión de la educación y la política de industrialización fueron algunos de los logros del gobierno de coalición que resultó del Pacto de Punto Fijo, indicando también el comienzo de un cierto consenso sobre tipos de políticas por seguir. Nada desdeñable tampoco fue la creación y consolidación de nuevas clientelas para los partidos que participaron en la coalición, especialmente para COPEI, que fue el único que se mantuvo hasta el final del periodo de Betancourt.

Esta experiencia en el gobierno incluyo los beneficios del patronazgo, especialmente en los tres ministerios encabezados por copeyanos. Se logro construir una fuerza electoral en áreas donde anteriormente no existía, a la vez que aumento su poder en los sindicatos. 9

\section{A pesar de que ha sido el Estado (basándose en su capacidad}

7 . Daniel Levine, "Venezuelan Politics: Past and Future", en Robert D. Bond (ed.), Contemporany Venezuela and its Role in International Affairs, Nueva York, New York University Press, 1977, p. 11.

8 Citado por José Antonio Gil Yepez, El reto de las elites, Madrid, Editorial Tecnos, 1978 , p. 5.

$9-$ John D. Martz, "The Campaign, Results and Interpretation" en Richard Fajen y Wayne Cornelius (eds.) Political Power in Latin America: Seven confrontations, Englewojd Clifs, N.J., Prentice-Hall, 1970, p. 62-71. p. 56. 
económica proveniente de la renta petrolera) quien ha proporcionado las prebendas económicas y sociales que garantizan la adhesión de las masas al sistema político, los grandes ganadores han sido los partidos políticos, especialemte los del status, pero tampoco se han excluido los otros. Todos tienen su cuota de representación y, por ende, de patronazgo, debido a la importancia de los contactos personales, de la omnipresente "palanca" en el funcionamiento del sistema.

En otras palabras, los sectores populares no se vinculan directamente con el Estado sino por intermedio de los partidos políticos. Los partidos políticos venezolanos se caracterizan por ser una mezcla de lo que hemos llamado el clientelismo corporativo y el clientelismo tradicional. Blank describe bastante bien este sistema doble de clientelismo. La mayoría de los venezolanos, según este autor, se relacionan en forma indirecta con el sistema partidista, a través de uno de dos tipos de intermediarios:

1. El cacique local, que perpetúa el clientelismo tradicional y que representa los intereses de sus clientes en forma relativamente poco diferenciada.

2. El líder político de las organizaciones funcionales más especializadas. Sin embargo, hay que destacar que aquí también en gran parte sigue funcionando la relación clientelista tradicional, en el sentido de favorecer los intereses particulares de sus miembros y no una categoría social como tal.

Aunque es de todos conocido, vale la pena también resaltar la naturaleza "cautiva" de las organizaciones de los sectores populares y una gran parte de las organizaciones de la clase media, que por su dependencia de los partidos muestran muy poca autonomía. Aquí sí entra con más propiedad el concepto de clientelismo corportivo, donde las peticiones corporativas o grupales tienen cabida, aunque no sean las únicas.

Debido tal vez a lo reciente del experimento democrático y a la poca legitimación del mismo y al mismo tiempo, la relativa abundancia de recursos a disposición del Estado, ha prevalecido el aspecto de intercambio en las relaciones clientelistas, mientras ha sido mucho más sutil el aspecto de control. Pero el hecho de haber sido más sutil no niega su importancia. Al contrario, el tipo de clientelismo que se ha desarrollado fortalece el control que los partidos políticos pueden tener sobre el nivel de conflicto político. En vez de congregar intereses (función generalmente atribuida a los partidos políticos), da la impresión de que los partidos venezolanos disgregan intereses. Por ejemplo, la contienda electoral, que podría ser el foro para la congregación de intereses en una forma relativamente peligrosa para el sistema político, los disgrega, diluyéndolos entre una multitud de pequeñas redes clientelistas que atienden más bien a intereses individualistas.

Por supuesto que esto no niega el conflicto, a veces violento, que obviamente existe entre los partidos políticos venezolanos, sobre todo entre los dos más importantes. Pero sí ayuda a explicar la escasez de contenido sustantivo en las campañas electorales, la tardanza en la presentación de los programas de gobierno, etcétera.

No hay que olvidar, para terminar esta sección, que los partidos políticos no son los únicos, y a veces ni siquiera los más importantes actores políticos. Existen otros actores o contenedores cuyo poder no tiene nada que ver con el apoyo popular, como son los sectores externo, indus-

10 David Eugene Blank, Politics in Venezuela, Boston, Little, Brown and Co., 1973, p. 98-101. 
trial, militar, eclesiástico, etc. Blank dice que desde 1958, "los partidos políticos han sido los actores cruciales en el establecimiento de la democracia representativa en Venezuela; pero han sido relativamente poco importantes en la determinación de las políticas económicas del gobierno". Tal vez los ejemplos más patentes de lo acertado de este comentario ocurrieron varios años después de que Blank escribiera su libro, durante las administraciones de Carlos Andrés Peréz y Luis Herrera Campins, sobre todo en la última, cuando las tensiones entre gobierno y partido de gobierno alcanzaron niveles tales que a veces parecía que el partido de gobierno estaba en la oposición. Hay ciertas indicaciones, en la renuncia de Luis Raúl Matos Azócar como ministro de Estado de Cordiplan, de que el mismo fenómeno comenzó a surgir en el gobierno de Jaime Lusinchi, a escasos once meses de la toma de poder.

Con estas breves consideraciones sobre el papel del clientelismo político en la conformación del modelo político venezolano, creemos que se enriquece el modelo propuesto por Levine para explicar la supervivencia del sistema político venezolano y su estabilidad lograda desde 1958. Levine propone cuatro variables cruciales para el éxito de ese modelo: 1) organizaciones poderosas y lealtad popular hacia ellas; 2) liderazgo autónomo y efectivo; 3 ) condiciones económicas favorables; y 4) una peculiar configuración de la escena internacional. Sin embargo, parece que Levine considera estas variables aisladamente, mientras que para nosotros parece obvio que la variable clientelista subyace en las primeras tres. Las condiciones economicas favorables permiten la creación de lazos clientelistas masivos, proporcionando la base de poder a las estructuras partidistas y manteniendo la lealtad popular hacia ellas. A su vez, el liderazgo autónomo y efectivo que permite la reconciliación y negociación entre elites que caracteriza al sistema, proviene del nivel de control logrado sobre las demandas populares a través de las bases clientelistas de los partidos.

En resumen, el modelo político venezolano instalado en 1958 se ha basado en la capacidad económica del Estado de subsidiar la democracia, proporcionando prebendas a todos los sectores importantes para mantener un cierto consenso social alrededor del sistema político. Tal vez la duración de ese modelo se deba más a los partidos que a ningún otro de los agentes políticos, pues han incorporado nuevos sectores populares al sistema mediante la creación de enormes redes clientelistas, compuestas por una multiplicidad de redecillas, en su mayor parte más de tipo tradicional que corporativo, proporcionando de esta forma una amplia base popular al sistema político, al mismo tiempo que dirimiendo los niveles de conflicto que pudieran surgir de la competencia electoral. Esto ha proporcionado cierta representatividad al sistema a la vez que permite que los otros contenedores por el poder tengan acceso a la formulación de políticas y que éstas se decidan en otros niveles menos parciales.

Pero la posibilidad de diluir los conflictos y el consiguiente control que sobre ellos puedan ejercer los partidos depende en gran parte del tipo de clientelismo político que han desarrollado y fomentado. A su vez, este tipo de clientelismo depende de una siempre creciente capacidad económica del Estado. En los últimos años, sin embargo, esta capacidad se ha visto amenazada, por un lado por la súbita rebaja en los precios del petróleo y, por otro, por la enormidad de la deuda externa nacional. ¿Cuáles podrán ser las posibles tendencias al cambio de este modelo, a la luz de la crisis financiera actual? 
En primer lugar, ya que el clientelismo político es una respuesta a condiciones de escasez de recursos, desigualmente distribuidos, y de inseguridad, se podría esperar que el clientelismo incrementara su importancia, en lugar de disminuirla. Esto significa que el modelo político probablemente seguirá siendo un modelo clientelista, pero no necesariamente significa que seguirá siendo el mismo que ha privado hasta ahora. Basándonos en una previa revisión teórica del clientelismo y en algunas comparaciones implícitas con México (que presenta rasgos del corporativo en forma más nítida), podemos adelantar algunas posibles tendencias al cambio.

Todas estas tendencias se pueden resumir en un énfasis mucho más marcado en el aspecto de control sobre las actividades de la clientela y en una disminución de la importancia del intercambio como elemento básico de la relación clientelista. Esta tendencia sería de esperar en la dinámica natural del desarrollo del clientelismo y más aún en una situación de crisis.

Existen varias posibilidades de lograr este mayor control con el régimen actual, sin que se recurra a la represión generalizada. Una primera sería reducir la dependencia de los partidos de las pequeñas redes clientelistas, que dependen para su eficacia de relaciones personales y de la satisfacción de múltiples intereses particulares y que requieren muchos recursos para funcionar.

Pero para impedir una eficaz congregación de intereses y dirimir el conflicto social, que habían sido las funciones principales de estas redes, habría que aumentar la adhesión de grupos funcionales o corporativos a las organizaciones cautivas de los partidos e incrementar el control partidista sobre ellas. Tal vez es precisamente un esfuerzo de este tipo el que subyace en las declaraciones del presidente Lusinchi y su apoyo por otros líderes partidistas, en el sentido de rechazar, casi como desleales, las presiones de la CTV. En este sentido, no parecería muy factible en este momento la democratización de los partidos ni la reforma electoral, que tanto se ha pregonado en la prensa venezolana.

Finalmente, parece factible que el Estado mismo intente institucionalizar el clientelismo político, vinculando las organizaciones funcionales de los sectores medios y populares directamente al Estado, mediante comisiones o consejos donde el Estado puede tener una voz decisiva o mediante regulaciones que vinculen a esos grupos a organismos burocráticos estatales. Este deseo parece implícito en la formulación del Pacto Social, base de la campaña electoral del ahora presidente, aunque sus primeras experiencias con la Comisión Nacional de Costos, Precios y Salarios (CONACOPRESA) no serían de muy buen augurio.

En conclusión, las posibilidades de una mayor democratización del modelo político venezolano en esta circunstancia no parecen ser muy prometedoras. Como tampoco la erradicación del clientelismo político, que se ha mostrado mucho más flexible y funcional de lo que quieren creer aquellos que lo han atacado como la causa de muchos males que padece el sistema político venezolano y que ven en la crisis financiera su fin. 


\section{BIBLIOGRAFIA}

Adams, Richard N., The Second Sowing: Power and Secondary Development in Latin America, San Francisco, Chandler, 1967, 288 p.

Alexander, Robert, "Democracy in Venezuela", LatinAmerican Research Review, vol. XV, núm. 2, 1980, p. 241-247.

Baloyra, Enrique A., "Criticism, Cynicism, and Political Evaluation: A Venezuelan Example", American Political Science Review, vol. 73, núm. 4, dic. 1979, p. 987-1002.

, Martz, John, Political Attitudes in Venezuela: Societal Cleavage and Po-

litical Opinion, Austin, University of Texas Press, 1979, $300 \mathrm{p}$.

Bautista Urbaneja, Diego, "El sistema político o cómo funciona la máquina de procesar decisiones", en M. Naim y R. Pif́ango (eds.), El caso Venezuela: una ilusión de armonía, Caracas, Ediciones IESA, 1984, p. 228-257.

Blank, David Eugene, Politics in Venezuela, Boston, Little, Brown and Co., 1973, 293 p.

Bonilla, Frank, El fracaso de las elites: cambio poltitico en Venezuela, vol. II, Caracas, Centro de Estudios del Desarrollo, Universidad Central de Venezuela, $1972,391 \mathrm{p}$.

Carrera Damas, Germán, Una nación llamada Venezuela: proceso sociohistórico de Venezuela (1810-1974), Caracas, Monte Avila Editores, 1984, 220 p.

Foster, George M., "The Dyadic Contract: A Model for the Social Structure of a Mexican Peasant Village", en S. Schmidt, J. Scott, C. Landé y L. Guasti (eds.), Friends, Followers and Factions, Berkeley, University of California Press, 1977, p. 15-28.

Gil Yepez, Jose Antonio, El reto de las elites, Madrid, Editorial Tecnos, 1978, p. 292.

Gouldner, Alvin W., "The Norm of Reciprocity: A Preliminary Statement", en S. Schmidt, J. Scott, C. Lande y L. Guasti (eds.), Friends, Followers and Factions, Berkeley, University of California Press, 1977, p. 288-293.

Guasti, Laura, "Perú: Clientelism and Internal Control", en S. Schmidt, J. Scott, C. Landé y L. Guasti (eds.), Friends, Followers and Factions, Berkeley, University of California Press, 1977, p. 422-438.

Hanes de Acevedo, Rexene A., El clientelismo pclitico en América Latina: una critica a la teoría de la dependencia, Mérida, Venezuela, Ediciones Librería Universitaria/Cruz del Sur, 1984, 190 p.

Lande, Carl H., "The Dyadic Basis of Clientelism", en S. Schmidt, J. Scott, C. Lande y L. Guasti (eds.), Friends, Followers and Factions, Berkeley, University of California Press, 1977, p. XIII-XXXVII.

Lemarchand, René y Keith Legg, "Political Clientelism and Development" Comparative Politics, vol. 44, núm. 2, enero 1972, p. 149-178.

Levine, Daniel, "Venezuelan Politics: Past and Future", en Robert D. Bond (ed.), Contemporary Venezuela and its Role in International Affairs, Nueva York, New York University Press, 1977, p. 7-44.

Martz, John D., "The Campaign, Results, and Interpretation", en Richard Fagen y Wayne Cornelius (eds.), Political Power in Latin America: Seven Confrontations, Englewood Cliffs, N.J, Prentice-Hall, 1970, p. 62-71.

Mintz, Sidney W., y Eric R., Wolf, "An Analysis of Ritual Co-Parenthood (Compadrazgo), en S. Schmidt, J. Scott, C. Lande y L. Guasti (eds.), Friends, Followers, and Factions, Berkeley, University of California Press, 1977, p. 1-15.

Naim, Moisés y Ramón Pifrango (eds.), El caso de Venezuela: una ilusión de armonía, Caracas, Ediciones IESA, 1984, 579 p.

Powell, John Duncan, "Peasant Society and Clientelist Politics", American Political Science Review, vol. 64, núm. 2, 1970, p. 411-425.

Sandbrook, Richard, "Patrons, Clients, and Factions: New Dimensions of Conflict Analysis in Africa", Canadian Journal of Political Science, vol. 5, núm. 2, marzo 1972, p. 104-119.

Schmidt, Steffen, James C. Scott, Carl Landé y Laura Guasti (eds.), Friends, Fo- 
llowers and Factions: A Reader in Political Clientelism, Berkeley, University of California Press, 1977,512 p.

Scott, James C., "Patron-Client Politics and Political Change, in Southeast Asia", American Political Science Review, vol. 66, núm. 1, marzo 1972, p. 91-113.

Sonntag, Heinz R., "Las elecciones en Venezuela: una interpretación inicial", en Nueva Sociedad, núm. 70, enero-febrero 1984, p. 136-146.

Suzzarini Baloa, Manuel, Rómulo Betancourt: proyecto de modernización, Caracas, Editorial Ateneo, 1981, 148 p.

Velázquez, Ramón J., "Proyección histórica de la obra de Rómulo Betancourt", en R. J. Velázquez, J. F. Sucre Figarella y Blas Bruni Celli, Betancourt en la Historia de Venezuela del siglo XX, Caracas, Ediciones Centauro, 1980,p. 5133.

Wolf, Eric, "Kinship, Friendship, and Patron-Ciient Relations in Complex Societies", en S. Schmidt, J. Scott, C. Lande y L. Guasti (eds.) Friends, Followers and Factions, Berkeley, University of California Press, 1977, p. 167-177. 